\title{
Estimates of the gluon concentrations in the confining SU(3)-Yang-Mills field for the first three states of charmonium
}

\author{
Yu. P. Goncharov \\ Theoretical Group, Experimental Physics Department, State Polytechnical \\ University, Sankt-Petersburg 195251, Russia \\ A. A. Bytsenko \\ Departamento de Fisica, Universidade Estadual de Londrina, Caixa Postal 6001, \\ Londrina-Parana, Brazil
}

\begin{abstract}
The estimates of the gluon concentrations in the classical SU(3)-Yang-Mills field modelling confinement are given for the first three states of charmonium whose spectrum is tuned by calculating electromagnetic transitions among the mentioned levels in dipole approximation. For comparison the corresponding estimates for the photon concentration in the ground state of positronium (parapositronium and orthopositronium) are also adduced.
\end{abstract}

Key words: quantum chromodynamics, confinement, charmonium PACS: 12.38.-t, 13.20.Gd, 14.40.Gx

\section{Introduction and preliminary remarks}

As was discussed in Refs. [1-3], the natural way of building meson spectroscopy might be based on the exact solutions of the SU(3)-Yang-Mills equations modelling quark confinement, the so-called confining solutions. Such solutions are supposed to contain only the components of the $\mathrm{SU}(3)$-field which are Coulomb-like or linear in $r$, the distance between quarks. In Ref. [1] a number of such solutions have been obtained and the corresponding spectrum of Dirac equation describing the relativistic bound states in those confining SU(3)-Yang-Mills fields has been analysed. It should be noted that the given 
approach is the direct consequence of the relativistic QCD (quantum chromodynamics) Lagrangian since the mentioned Yang-Mills and Dirac equations are derived just from the latter one.

Further in Refs. $[2,3]$ the results obtained were successfully applied to the description of the quarkonia spectra (charmonium and bottomonium). In its turn, the mentioned description points out the linear confinement to be (classically) governed by the colour magnetic field linear in $r$.

The results of Refs. [1-3] suggest the following mechanism of confinement to occur within the framework of QCD (at any rate, for mesons and quarkonia). The gluon exchange between quarks is realized in such a way that at large distances it leads to the confining SU(3)-field which may be considered classically (the gluon concentration becomes huge and gluons form the boson condensate - a classical field) and is a nonperturbative solution of the SU(3)-Yang-Mills equations. Under the circumstances mesons are the relativistic bound states described by the corresponding wave functions - nonperturbative solutions of the Dirac equation in this confining $\mathrm{SU}(3)$-field [1-3]. For each meson there exists its own set of real constants (for more details see below) $a_{j}, A_{j}, b_{j}, B_{j}$ parametrizing the mentioned confining gluon field (the gluon condensate) and the corresponding wave functions while the latter ones also depend on $\mu_{0}$, the reduced mass of the current masses of quarks forming meson [1-3]. It is clear that constants $a_{j}, A_{j}, b_{j}, B_{j}, \mu_{0}$ should be extracted from experimental data and such a program has been just realized in Refs. [2,3] for quarkonia.

The aim of the present paper is to estimate the above gluon concentrations for the case of charmonium but we shall restrict ourselves to the first three states of charmonium. For comparison we shall also adduce the corresponding estimates for the photon concentrations in the ground state of positronium (parapositronium and orthopositronium) to emphasize some analogy between QCD and QED (quantum electrodynamics).

Further we shall deal with the metric of the flat Minkowski spacetime $M$ that we write down (using the ordinary set of local spherical coordinates $r, \vartheta, \varphi$ for the spatial part) in the form

$$
d s^{2}=g_{\mu \nu} d x^{\mu} \otimes d x^{\nu} \equiv d t^{2}-d r^{2}-r^{2}\left(d \vartheta^{2}+\sin ^{2} \vartheta d \varphi^{2}\right) .
$$

Besides, we have $|\delta|=\left|\operatorname{det}\left(g_{\mu \nu}\right)\right|=\left(r^{2} \sin \vartheta\right)^{2}$ and $0 \leq r<\infty, 0 \leq \vartheta<\pi$, $0 \leq \varphi<2 \pi$.

Throughout the paper we employ the system of units with $\hbar=c=1$, unless explicitly stated otherwise. Finally, we shall denote $L_{2}(F)$ the set of the modulo square integrable complex functions on any manifold $F$ furnished with an integration measure while $L_{2}^{n}(F)$ will be the $n$-fold direct product of $L_{2}(F)$ endowed with the obvious scalar product. 
If $A=A_{\mu} d x^{\mu}=A_{\mu}^{c} \lambda_{c} d x^{\mu}$ is a $\mathrm{SU}(3)$-connection in the (trivial) three-dimensional bundle $\xi$ over the Minkowski spacetime, where $\lambda_{c}$ are the known Gell-Mann matrices, then we are interested in the confining solutions $A$ of the $\mathrm{SU}(3)$ Yang-Mills equations

$$
d * F=g(* F \wedge A-A \wedge * F)
$$

with the exterior differential $d=\partial_{t} d t+\partial_{r} d r+\partial_{\vartheta} d \vartheta+\partial_{\varphi} d \varphi$ in coordinates $t, r, \vartheta, \varphi$, while the curvature matrix (field strentgh) for the $\xi$-bundle is $F=$ $d A+g A \wedge A=F_{\mu \nu}^{a} \lambda_{a} d x^{\mu} \wedge d x^{\nu}$ and $*$ means the Hodge star operator conforming to metric (1), $g$ is a gauge coupling constant.

For the aims of the given paper we shall use the confining solution of Ref. [1] in the form

$$
\begin{aligned}
& A_{t}^{3}+\frac{1}{\sqrt{3}} A_{t}^{8}=-\frac{a_{1}}{r}+A_{1},-A_{t}^{3}+\frac{1}{\sqrt{3}} A_{t}^{8}=\frac{a_{1}+a_{2}}{r}-\left(A_{1}+A_{2}\right),-\frac{2}{\sqrt{3}} A_{t}^{8}=-\frac{a_{2}}{r}+A_{2}, \\
& A_{\varphi}^{3}+\frac{1}{\sqrt{3}} A_{\varphi}^{8}=b_{1} r+B_{1},-A_{\varphi}^{3}+\frac{1}{\sqrt{3}} A_{\varphi}^{8}=-\left(b_{1}+b_{2}\right) r-\left(B_{1}+B_{2}\right),-\frac{2}{\sqrt{3}} A_{\varphi}^{8}=b_{2} r+B_{2}
\end{aligned}
$$

with all other $A_{\mu}^{c}=0$, where real constants $a_{j}, A_{j}, b_{j}, B_{j}$ parametrize the solution, and we wrote down the solution in the combinations that are just needed to insert into the Dirac equation

$$
\mathcal{D} \Psi=\mu_{0} \Psi
$$

with the relativistic wave function $\Psi=\left(\Psi_{1}, \Psi_{2}, \Psi_{3}\right)$ of the quarkonium, where the four-dimensional spinors $\Psi_{j}$ represent the $j$-th colour component of the quarkonium, $\mu_{0}$ is a mass parameter and one can consider it to be the reduced relativistic mass which is equal, e. $g$. for quarkonia, to half the current mass of quarks forming a quarkonium, while the coordinate $r$ stands for the distance between quarks. The explicit form of operator $\mathcal{D}$ is not needed here and can be found in Refs. [1-3].

Finally, for the necessary estimates we shall employ the $T_{00}$-component of the energy-momentum tensor for a SU(3)-Yang-Mills field

$$
T_{\mu \nu}=\frac{1}{4 \pi}\left(-F_{\mu \alpha}^{a} F_{\nu \beta}^{a} g^{\alpha \beta}+\frac{1}{4} F_{\beta \gamma}^{a} F_{\alpha \delta}^{a} g^{\alpha \beta} g^{\gamma \delta} g_{\mu \nu}\right)
$$

\section{Relativistic spectrum of charmonium}

From the adduced form it is clear that the solution (3) is a configuration describing the electric Coulomb-like colour field (components $A_{t}$ ) and the magnetic colour field linear in $r$ (components $A_{\varphi}$ ) so the solution can be rewritten 
as

$$
\begin{gathered}
A_{t}^{3}=-\left(a_{1}+a_{2} / 2\right) / r+A_{1}+A_{2} / 2, A_{t}^{8}=\left(a_{2} / r-A_{2}\right) \sqrt{3} / 2, \\
A_{\varphi}^{3}=\left(b_{1}+b_{2} / 2\right) r+B_{1}+B_{2} / 2, A_{\varphi}^{8}=-\left(b_{2} r+B_{2}\right) \sqrt{3} / 2 .
\end{gathered}
$$

As was shown in Ref. [1], after inserting the above confining solution into Eq. (4), it admits the solutions of the form (with Pauli matrix $\sigma_{1}$ )

$$
\Psi_{j}=e^{i \omega_{j} t} r^{-1}\left(\begin{array}{c}
F_{j 1}(r) \Phi_{j}(\vartheta, \varphi) \\
F_{j 2}(r) \sigma_{1} \Phi_{j}(\vartheta, \varphi)
\end{array}\right), j=1,2,3
$$

with the $2 \mathrm{D}$ eigenspinor $\Phi_{j}=\left(\begin{array}{l}\Phi_{j 1} \\ \Phi_{j 2}\end{array}\right)$ of the euclidean Dirac operator on the unit sphere $\mathbb{S}^{2}$. The general explicit form of $\Phi_{j}$ is not needed here and can be found in Refs. [4]. For the purpose of the present paper we shall adduce the necessary spinors below. Spinors $\Phi_{j}$ form an orthonormal basis in $L_{2}^{2}\left(\mathbb{S}^{2}\right)$ and they can be subject to the normalization condition

$$
\int_{\mathbb{S}^{2}} \Phi_{j}^{\dagger} \Phi_{j} d \Omega=\int_{0}^{\pi} \int_{0}^{2 \pi}\left(\left|\Phi_{j 1}\right|^{2}+\left|\Phi_{j 2}\right|^{2}\right) \sin \vartheta d \vartheta d \varphi=1
$$

where $(\dagger)$ stands for hermitian conjugation.

The energy spectrum $\varepsilon$ of quarkonium is given by the relation $\varepsilon=\omega_{1}+\omega_{2}+\omega_{3}$ with

$$
\begin{gathered}
\omega_{1}=\omega_{1}\left(n_{1}, l_{1}, \lambda_{1}\right)=g A_{1}+\frac{-\Lambda_{1} g^{2} a_{1} b_{1}+\left(n_{1}+\alpha_{1}\right) \sqrt{\left(n_{1}^{2}+2 n_{1} \alpha_{1}+\Lambda_{1}^{2}\right) \mu_{0}^{2}+g^{2} b_{1}^{2}\left(n_{1}^{2}+2 n_{1} \alpha_{1}\right)}}{n_{1}^{2}+2 n_{1} \alpha_{1}+\Lambda_{1}^{2}}, \\
\begin{array}{c}
\omega_{2}=\omega_{2}\left(n_{2}, l_{2}, \lambda_{2}\right)=-g\left(A_{1}+A_{2}\right)+ \\
n_{2}^{2}+2 n_{2} \alpha_{2}+\Lambda_{2}^{2}
\end{array} \\
\omega_{3}=\omega_{3}\left(n_{3}, l_{3}, \lambda_{3}\right)\left(b_{1}+b_{2}\right)-\left(n_{2}+\alpha_{2}\right) \sqrt{\left(n_{2}^{2}+2 n_{2} \alpha_{2}+\Lambda_{2}^{2}\right) \mu_{0}^{2}+g^{2}\left(b_{1}+b_{2}\right)^{2}\left(n_{2}^{2}+2 n_{2} \alpha_{2}\right)} \\
n_{2}+\frac{-\Lambda_{3} g^{2} a_{2} b_{2}+\left(n_{3}+\alpha_{3}\right) \sqrt{\left(n_{3}^{2}+2 n_{3} \alpha_{3}+\Lambda_{3}^{2}\right) \mu_{0}^{2}+g^{2} b_{2}^{2}\left(n_{3}^{2}+2 n_{3} \alpha_{3}\right)}}{n_{3}^{2}+2 n_{3} \alpha_{3}+\Lambda_{3}^{2}}
\end{gathered}
$$

where $\Lambda_{1}=\lambda_{1}-g B_{1}, \Lambda_{2}=\lambda_{2}+g\left(B_{1}+B_{2}\right), \Lambda_{3}=\lambda_{3}-g B_{2}, n_{j}=0,1,2, \ldots$, while $\lambda_{j}= \pm\left(l_{j}+1\right)$ are the eigenvalues of euclidean Dirac operator on unit sphere with $l_{j}=0,1,2, \ldots$ Besides

$$
\alpha_{1}=\sqrt{\Lambda_{1}^{2}-g^{2} a_{1}^{2}}, \alpha_{2}=\sqrt{\Lambda_{2}^{2}-g^{2}\left(a_{1}+a_{2}\right)^{2}}, \alpha_{3}=\sqrt{\Lambda_{3}^{2}-g^{2} a_{2}^{2}} .
$$

Further, the radial parts of (7) are given at $n_{j}=0$ by

$$
F_{j 1}=C_{j} P_{j} r^{\alpha_{j}} e^{-\beta_{j} r}\left(1-\frac{Y_{j}}{Z_{j}}\right), F_{j 2}=i C_{j} Q_{j} r^{\alpha_{j}} e^{-\beta_{j} r}\left(1+\frac{Y_{j}}{Z_{j}}\right),
$$


Table 1

Gauge coupling constant, mass parameter $\mu_{0}$ and parameters of the confining SU(3)connection for charmonium.

$\begin{array}{llllllll}\mu_{0} & a_{1} & a_{2} & b_{1} & b_{2} & B_{1} & B_{2}\end{array}$

$(\mathrm{GeV}) \quad(\mathrm{GeV}) \quad(\mathrm{GeV})$

$\begin{array}{llllllll}0.46900 & 0.62500 & 2.21104 & -0.751317 & 20.2395 & -12.6317 & 6.89659 & 6.89659\end{array}$

while at $n_{j}>0$ by

$$
\begin{gathered}
F_{j 1}=C_{j} P_{j} r^{\alpha_{j}} e^{-\beta_{j} r}\left[\left(1-\frac{Y_{j}}{Z_{j}}\right) L_{n_{j}}^{2 \alpha_{j}}\left(r_{j}\right)+\frac{P_{j} Q_{j}}{Z_{j}} r_{j} L_{n_{j}-1}^{2 \alpha_{j}+1}\left(r_{j}\right)\right], \\
F_{j 2}=i C_{j} Q_{j} r^{\alpha_{j}} e^{-\beta_{j} r}\left[\left(1+\frac{Y_{j}}{Z_{j}}\right) L_{n_{j}}^{2 \alpha_{j}}\left(r_{j}\right)-\frac{P_{j} Q_{j}}{Z_{j}} r_{j} L_{n_{j}-1}^{2 \alpha_{j}+1}\left(r_{j}\right)\right],
\end{gathered}
$$

with the Laguerre polynomials $L_{n_{j}}^{\rho}\left(r_{j}\right), r_{j}=2 \beta_{j} r$, where, for $j=1, \beta_{1}=$ $\sqrt{\mu_{0}^{2}-\left(\omega_{1}-g A_{1}\right)^{2}+g^{2} b_{1}^{2}}, P_{1}=g b_{1}+\beta_{1}, Q_{1}=\mu_{0}+\omega_{1}-g A_{1}, Y_{1}=\left[\alpha_{1} \beta_{1}-\right.$ $\left.\left.g a_{1}\left(\omega_{1}-g A_{1}\right)+g \alpha_{1} b_{1}\right] Q_{1}+g^{2} a_{1} b_{1} P_{1}, Z_{1}=\left[\left(\lambda_{1}-g B_{1}\right) P_{1}+g a_{1} \mu_{0}\right)\right] Q_{1}+g^{2} a_{1} b_{1} P_{1}$, while for $j=2$ one should replace $a_{1}, A_{1}, b_{1}, B_{1} \rightarrow-\left(a_{1}+a_{2}\right),-\left(A_{1}+\right.$ $\left.A_{2}\right),-\left(b_{1}+b_{2}\right),-\left(B_{1}+B_{2}\right)$ and for $j=3$ one should substitute $a_{1}, A_{1}, b_{1}, B_{1}$ for $a_{2}, A_{2}, b_{2}, B_{2}$ to obtain the corresponding quantities $P_{j}, Q_{j}, Y_{j}, Z_{j}$, so that, for instance, $\beta_{2}=\sqrt{\mu_{0}^{2}-\left[\omega_{2}+g\left(A_{1}+A_{2}\right)\right]^{2}+g^{2}\left(b_{1}+b_{2}\right)^{2}}, \beta_{3}=\sqrt{\mu_{0}^{2}-\left(\omega_{3}-g A_{2}\right)^{2}+g^{2} b_{2}^{2}}$. Also it should be noted that the quantum numbers $n_{j}$ of (9)-(11) are defined by the relations (for more details see Ref. [1])

$$
n_{1}=\frac{g b_{1} Z_{1}-\beta_{1} Y_{1}}{\beta_{1} P_{1} Q_{1}}, n_{2}=-\frac{g\left(b_{1}+b_{2}\right) Z_{2}+\beta_{2} Y_{2}}{\beta_{2} P_{2} Q_{2}}, n_{3}=\frac{g b_{2} Z_{3}-\beta_{3} Y_{3}}{\beta_{3} P_{3} Q_{3}} .
$$

Finally, $C_{j}$ is determined from the normalization condition

$$
\int_{0}^{\infty}\left(\left|F_{j 1}\right|^{2}+\left|F_{j 2}\right|^{2}\right) d r=\frac{1}{3} .
$$

Consequently, we shall gain that $\Psi_{j} \in L_{2}^{4}\left(\mathbb{R}^{3}\right)$ at any $t \in \mathbb{R}$ and, as a result, the solutions of (7) may describe relativistic bound states of quarkonium with the energy spectrum (9)-(11).

Now we can adduce numerical results for constants parametrizing the charmonium spectrum which are shown in Table 1.

One can note that the obtained mass parameter $\mu_{0}$ is consistent with the present-day experimental limits [5] where the current mass of $c$-quark $\left(2 \mu_{0}\right)$ is 
Table 2

Theoretical and experimental charmonium levels.

\begin{tabular}{c|c|c}
\hline State & Theoret. energy $\varepsilon_{j}=\sum_{k=1}^{3} \omega_{k}$ & Experim. energy value \\
& $(\mathrm{GeV})$ & $(\mathrm{GeV})$ \\
\hline$\eta_{c}(1 S)$ & $\varepsilon_{1}=\omega_{1}(0,0,-1)+\omega_{2}(0,0,-1)+\omega_{3}(0,0,-1)=2.979597$ & 2.979600 \\
\hline$J / \psi(1 S)$ & $\varepsilon_{2}=\omega_{1}(0,0,-1)+\omega_{2}(0,0,1)+\omega_{3}(0,0,-1)=3.096913$ & 3.096916 \\
\hline$\chi_{c 0}(1 P)$ & $\varepsilon_{3}=\omega_{1}(0,0,-1)+\omega_{2}(0,0,-1)+\omega_{3}(0,0,1)=3.415186$ & 3.415190 \\
\hline$\chi_{c 1}(1 P)$ & $\varepsilon_{4}=\omega_{1}(0,0,1)+\omega_{2}(2,0,1)+\omega_{3}(0,1,-1)=3.505304$ & 3.510590 \\
\hline$h_{c}(1 P)$ & $\varepsilon_{5}=\omega_{1}(0,0,-1)+\omega_{2}(0,0,1)+\omega_{3}(0,0,1)=3.532503$ & 3.526210 \\
\hline$\chi_{c 2}(1 P)$ & $\varepsilon_{6}=\omega_{1}(0,1,-1)+\omega_{2}(1,1,-1)+\omega_{3}(1,1,-1)=3.553097$ & 3.556260 \\
\hline$\eta_{c}(2 S)$ & $\varepsilon_{7}=\omega_{1}(0,0,1)+\omega_{2}(1,0,-1)+\omega_{3}(0,1,-1)=3.671608$ & 3.65400 \\
\hline$\psi(2 S)$ & $\varepsilon_{8}=\omega_{1}(0,1,-1)+\omega_{2}(2,1,1)+\omega_{3}(1,1,-1)=3.674025$ & 3.685093 \\
\hline$\psi(3770)$ & $\varepsilon_{9}=\omega_{1}(0,0,1)+\omega_{2}(2,0,-1)+\omega_{3}(0,0,1)=3.775598$ & 3.770000 \\
\hline$\psi(3836)$ & $\varepsilon_{10}=\omega_{1}(0,1,-1)+\omega_{2}(0,0,1)+\omega_{3}(0,1,1)=3.833640$ & 3.836000 \\
\hline$X(3872)$ & $\varepsilon_{11}=\omega_{1}(0,1,-1)+\omega_{2}(0,1,1)+\omega_{3}(0,1,1)=3.871672$ & 3.872000 \\
\hline$\psi(4040)$ & $\varepsilon_{12}=\omega_{1}(0,0,1)+\omega_{2}(1,1,1)+\omega_{3}(0,1,-1)=4.042660$ & 4.040000 \\
\hline$\psi(4160)$ & $\varepsilon_{13}=\omega_{1}(0,0,-1)+\omega_{2}(0,0,-1)+\omega_{3}(0,1,1)=4.153765$ & 4.159000 \\
\hline$\psi(4415)$ & $\varepsilon_{14}=\omega_{1}(0,0,-1)+\omega_{2}(2,1,1)+\omega_{3}(1,0,-1)=4.409260$ & 4.415000 \\
\hline
\end{tabular}

accepted between $1.1 \mathrm{GeV}$ and $1.4 \mathrm{GeV}$. As for the gauge coupling constant $g$ then its value has been chosen in accordance with many recent considerations [6], wherefrom one can conclude that the strong coupling constant $\alpha_{s}=g^{2}$ is of order $0.22 \approx 0.469^{2}$ at the scale of the $c$-quark current mass. At last, as to parameters $A_{1,2}$ of solution (3), it is clear that they only shift the origin of count for the corresponding energies and we can consider $A_{1}=A_{2}=0$.

With the constants of Table 1 the present-day levels of charmonium spectrum were calculated with the help of (9)-(11) so Table 2 contains experimental values of these levels (from Ref. [5]) and our theoretical ones computed according to the shown combinations (we use the notations of levels from Ref. $[5])$. 
3 Specification of spectrum: electromagnetic transitions $J / \psi(1 S) \rightarrow$ $\eta_{c}(1 S)+\gamma$ and $\chi_{c 0}(1 P) \rightarrow J / \psi(1 S)+\gamma$ in dipole approximation

Now we should specify the obtained above charmonium spectrum. The fact is that the relations (9)-(11) permit various parametrizations of the charmonium spectrum (see Refs. [2,3]) and therefore it should impose further conditions to fix a certain parametrization among several possible ones. In the present paper we shall restrict ourselves to elecromagnetic transitions $J / \psi(1 S) \rightarrow$ $\eta_{c}(1 S)+\gamma$ and $\chi_{c 0}(1 P) \rightarrow J / \psi(1 S)+\gamma$ since we are interested in the first three levels of charmonium. We shall compute widths of the mentioned transitions in dipole approximation that will allow us to use the corresponding wave functions written out in (13)-(14). Dipole approximation is often employed in meson physics, at least, as a primordial width estimate for one or another electromagnetic transition (see, e. g. Ref. [7]) so we shall, without going into details, remind that in dipole approximation a width $\Gamma$ of an electromagnetic transition with emission of one photon for some system is given by the relation (for more details see, e. g. Ref. [8])

$$
\Gamma=\frac{4 \omega^{3}}{3}\left|d_{f i}\right|^{2},
$$

where $d_{f i}-$ a matrix element of the system dipole moment $\mathbf{d}=Q(x \mathbf{i}+y \mathbf{j}+z \mathbf{k})$, $Q$ is an electromagnetic charge of system, the matrix element is taken between a final system stationary state and an initial one and $\omega$ is the transition frequency. Let us introduce the quantities $\xi=x+i y=r \sin \vartheta e^{i \varphi}, \eta=x-i y=$ $r \sin \vartheta e^{-i \varphi}, z=r \cos \vartheta$, then it is clear that

$$
\left|d_{f i}\right|^{2}=Q^{2}\left[\frac{\left|\xi_{f i}\right|^{2}+\left|\eta_{f i}\right|^{2}}{2}+\left|z_{f i}\right|^{2}\right],
$$

while in our case $Q^{2}=(2 e / 3)^{2}$ with $e^{2}=1 / 137.0359895$. We should compute the experimental widths $\Gamma\left(J / \psi(1 S) \rightarrow \eta_{c}(1 S)+\gamma\right)=\Gamma_{94}=1.1557 \mathrm{keV}$ and $\Gamma\left(\chi_{c 0}(1 P) \rightarrow J / \psi(1 S)+\gamma\right)=\Gamma_{16}=120.36 \mathrm{keV}$ (data and notations from [5]) with the transition frequencies $\omega_{94}=0.117316 \mathrm{GeV}, \omega_{16}=0.318274 \mathrm{GeV}$.

\subsection{Calculation of $\Gamma_{94}$}

As is clear from Table 2, the given transition is conditioned by the corresponding one of the second colour component $\omega_{2}(0,0,1) \rightarrow \omega_{2}(0,0,-1)$. The initial stationary wave function according to $(7)$ is

$$
\psi_{2}^{i}=r^{-1}\left(\begin{array}{c}
F_{21}^{i} \Phi_{2}^{i} \\
F_{22}^{i} \sigma_{1} \Phi_{2}^{i}
\end{array}\right)
$$


with the $2 \mathrm{D}$ eigenspinor $\Phi_{2}^{i}$ of the euclidean Dirac operator $\mathcal{D}_{2}$ on the unit sphere $\mathbb{S}^{2}$ conforming to the eigenvalue $\lambda=\lambda_{2}=1$ while the final stationary wave function is

$$
\psi_{2}^{f}=r^{-1}\left(\begin{array}{c}
F_{21}^{f} \Phi_{2}^{f} \\
F_{22}^{f} \sigma_{1} \Phi_{2}^{f}
\end{array}\right)
$$

with the $2 \mathrm{D}$ eigenspinor $\Phi_{2}^{f}$ of $\mathcal{D}_{2}$ conforming to the eigenvalue $\lambda=\lambda_{2}=-1$. Further we have $F_{21}^{i}=C_{2}^{i} P_{2}^{i} r^{\alpha_{2}^{i}} e^{-\beta_{2}^{i} r}\left[1+\frac{g\left(b_{1}+b_{2}\right)}{\beta_{2}^{i}}\right], F_{22}^{i}=i C_{2}^{i} Q_{2}^{i} r^{\alpha_{2}^{i}} e^{-\beta_{2}^{i} r}\left[1-\frac{g\left(b_{1}+b_{2}\right)}{\beta_{2}^{i}}\right]$ with $P_{2}^{i}=-g\left(b_{1}+b_{2}\right)+\beta_{2}^{i}, Q_{2}^{i}=\mu_{0}+\omega_{2}^{i}, \beta_{2}^{i}=\sqrt{\mu_{0}^{2}-\left(\omega_{2}^{i}\right)^{2}+g^{2}\left(b_{1}+b_{2}\right)^{2}}$, $\alpha_{2}^{i}=\sqrt{\left[1+g\left(B_{1}+B_{2}\right)\right]^{2}-g^{2}\left(a_{1}+a_{2}\right)^{2}}, \omega_{2}^{i}=\omega_{2}(0,0,1)$ as well as $F_{21}^{f}=$ $C_{2}^{f} P_{2}^{f} r^{\alpha_{2}^{f}} e^{-\beta_{2}^{f} r}\left[1+\frac{g\left(b_{1}+b_{2}\right)}{\beta_{2}^{f}}\right], F_{22}^{f}=i C_{2}^{f} Q_{2}^{f} r^{\alpha_{2}^{f}} e^{-\beta_{2}^{f} r}\left[1-\frac{g\left(b_{1}+b_{2}\right)}{\beta_{2}^{f}}\right]$ with $P_{2}^{f}=$ $-g\left(b_{1}+b_{2}\right)+\beta_{2}^{f}, Q_{2}^{f}=\mu_{0}+\omega_{2}^{f}, \beta_{2}^{f}=\sqrt{\mu_{0}^{2}-\left(\omega_{2}^{f}\right)^{2}+g^{2}\left(b_{1}+b_{2}\right)^{2}}, \alpha_{2}^{f}=$ $\sqrt{\left[-1+g\left(B_{1}+B_{2}\right)\right]^{2}-g^{2}\left(a_{1}+a_{2}\right)^{2}}, \omega_{2}^{f}=\omega_{2}(0,0,-1)$ and we took into account that according to (15) $Y_{2} / Z_{2}=-g\left(b_{1}+b_{2}\right) / \beta_{2}$ at $n_{2}=0$. Also for constant $C_{2}^{i}$ we obtain from (16) (with the help of formula [9] $\int_{0}^{\infty} x^{\alpha-1} e^{-p x} d x=$ $\left.\Gamma(\alpha) p^{-\alpha}, \operatorname{Re} \alpha, p>0\right)$

$$
\left(C_{2}^{i}\right)^{2}\left[\left(1+\frac{g\left(b_{1}+b_{2}\right)}{\beta_{2}^{i}}\right)^{2}\left(P_{2}^{i}\right)^{2}+\left(1-\frac{g\left(b_{1}+b_{2}\right)}{\beta_{2}^{i}}\right)^{2}\left(Q_{2}^{i}\right)^{2}\right] \frac{\Gamma\left(2 \alpha_{2}^{i}+1\right)}{\left(2 \beta_{2}^{i}\right)^{\left(2 \alpha_{2}^{i}+1\right)}}=\frac{1}{3}
$$

whereas the analogous relation for $C_{2}^{f}$ can be obtained from (21) by changing indices $i \rightarrow f$.

At last, as for spinors $\Phi_{2}^{i}, \Phi_{2}^{f}$, it should be noted that the eigenvalues of Dirac operator $\mathcal{D}_{2}$ have the multiplicity $2\left(l_{2}+1\right)$ for each $\lambda_{2}= \pm\left(l_{2}+1\right)$, so the spinors $\Phi_{2}^{i}, \Phi_{2}^{f}\left(l_{2}=0\right)$ can be taken in the forms (for more details see Refs. [4])

$$
\begin{gathered}
\Phi_{2}^{i 1}=\frac{C}{2}\left(\begin{array}{c}
\cos \frac{\vartheta}{2}+i \sin \frac{\vartheta}{2} \\
\cos \frac{\vartheta}{2}-i \sin \frac{\vartheta}{2}
\end{array}\right) e^{i \varphi / 2}, \Phi_{2}^{i 2}=\frac{C}{2}\left(\begin{array}{c}
\cos \frac{\vartheta}{2}+i \sin \frac{\vartheta}{2} \\
-\cos \frac{\vartheta}{2}+i \sin \frac{\vartheta}{2}
\end{array}\right) e^{-i \varphi / 2}, \\
\Phi_{2}^{f 1}=\frac{C}{2}\left(\begin{array}{l}
\cos \frac{\vartheta}{2}-i \sin \frac{\vartheta}{2} \\
\cos \frac{\vartheta}{2}+i \sin \frac{\vartheta}{2}
\end{array}\right) e^{i \varphi / 2}, \Phi_{2}^{f 2}=\frac{C}{2}\left(\begin{array}{c}
-\cos \frac{\vartheta}{2}+i \sin \frac{\vartheta}{2} \\
\cos \frac{\vartheta}{2}+i \sin \frac{\vartheta}{2}
\end{array}\right) e^{-i \varphi / 2},
\end{gathered}
$$

where the coefficient $C=\sqrt{1 /(2 \pi)}$.

Under the circumstances we shall have [(*) signifies complex conjugation]

$$
\xi_{f i}=\int\left(\psi_{2}^{f}\right)^{\dagger} \xi \psi_{2}^{i} d^{3} x=\int_{\mathbb{R}^{3}} r\left(F_{21}^{* f} F_{21}^{i}+F_{22}^{* f} F_{22}^{i}\right)\left[\sum\left(\Phi_{2}^{f}\right)^{\dagger} \Phi_{2}^{i}\right] \sin ^{2} \vartheta e^{i \varphi} d r d \vartheta d \varphi
$$

where $\sum\left(\Phi_{2}^{f}\right)^{\dagger} \Phi_{2}^{i}$ is the sum over all possible combinations of final and initial spinors. It is then not complicated to see that the only nonzero contribution comes from the combination $\left(\Phi_{2}^{f 1}\right)^{\dagger} \Phi_{2}^{i 2}=e^{-i \varphi}\left(i C^{2} \sin \vartheta\right) / 2$ while the rest of 
combinations give zero when integrating over $\varphi$ in (23). Fulfiling the remaining integration in (23) over $\vartheta$ and $r$ yields

$$
\begin{gathered}
\xi_{f i}=i \frac{2}{3} C_{2}^{i} C_{2}^{f} \frac{\Gamma\left(\alpha_{2}^{f}+\alpha_{2}^{i}+2\right)}{\left(\beta_{2}^{f}+\beta_{2}^{i}\right)^{\alpha_{2}^{f}+\alpha_{2}^{i}+2}} \times \\
\left\{P_{2}^{f} P_{2}^{i}\left[1+\frac{g\left(b_{1}+b_{2}\right)}{\beta_{2}^{f}}\right]\left[1+\frac{g\left(b_{1}+b_{2}\right)}{\beta_{2}^{i}}\right]+Q_{2}^{f} Q_{2}^{i}\left[1-\frac{g\left(b_{1}+b_{2}\right)}{\beta_{2}^{f}}\right]\left[1-\frac{g\left(b_{1}+b_{2}\right)}{\beta_{2}^{i}}\right]\right\} .
\end{gathered}
$$

The similar considerations show that $\eta_{f i} \neq 0$ only for the spinor combination $\left(\Phi_{2}^{f 2}\right)^{\dagger} \Phi_{2}^{i 1}=-e^{i \varphi}\left(i C^{2} \sin \vartheta\right) / 2$ which entails $\xi_{f i}=-\eta_{f i}$ while two spinor combinations giving the contributions to $z_{f i}$ are $\left(\Phi_{2}^{f 2}\right)^{\dagger} \Phi_{2}^{i 2}=-\left(\Phi_{2}^{f 1}\right)^{\dagger} \Phi_{2}^{i 1}=$ $\cos \vartheta /(8 \pi)$ which gives rise to $z_{f i}=0$. As a result, we obtain according to (18)

$$
\Gamma_{94}=\frac{4 \omega_{94}^{3}}{3} Q^{2}\left|\xi_{f i}\right|^{2}
$$

with $\xi_{f i}$ of $(24)$.

\subsection{Calculation of $\Gamma_{16}$}

In accordance with Table 2 the given transition is formed by two ones: the photon absorption with frequency $\omega_{I}=\omega_{2}(0,0,1)-\omega_{2}(0,0,-1)=0.117316$ $\mathrm{GeV}$ by the second colour component and the photon emission with frequency $\omega_{I I}=\omega_{3}(0,0,1)-\omega_{3}(0,0,-1)=0.435591 \mathrm{GeV}$ by the third colour component so the resulting frequency $\omega_{16}=\omega_{I I}-\omega_{I}=0.318275 \mathrm{GeV}$. Accordingly we can write $\Gamma_{16}=\Gamma_{I}+\Gamma_{I I}$. Now the considerations analogous to the above ones show that again $\xi_{f i}=-\eta_{f i}, z_{f i}=0$ in both transitions and $\Gamma_{I}$ is obtained from (24)-(25) by replacing $i \rightarrow f, f \rightarrow i, \omega_{94} \rightarrow \omega_{I}$ while $\Gamma_{I I}$ will be the result of changing indices $2 \rightarrow 3$ and $\left(b_{1}+b_{2}\right) \rightarrow-b_{2},\left(a_{1}+a_{2}\right) \rightarrow-a_{2}$, $\left(B_{1}+B_{2}\right) \rightarrow-B_{2}, \omega_{94} \rightarrow \omega_{I I}$ in $(24)-(25)$.

\subsection{Numerical results}

Table 3 gives the results of evaluation for $\Gamma_{94}$ and $\Gamma_{16}$ according to the obtained relations and the corresponding experimental ones of Ref. [5]. Table 3 supplies us with an additional justification for the choice of parameters of the SU(3)-confining gluon field adduced in Table 1 and allows us to conclude that dipole approximation is not enough for the second transition of the ones under consideration. The question now is what gluon concentrations are in the mentioned SU(3)-confining gluon field. 
Table 3

Widths of electromagnetic transitions in charmonium.

\begin{tabular}{c|c|c}
\hline$\Gamma$ & $\begin{array}{c}\text { Theoret. value } \\
(\mathrm{keV})\end{array}$ & $\begin{array}{c}\text { Experim. value } \\
(\mathrm{keV})\end{array}$ \\
\hline$\Gamma_{94}$ & 1.02977 & 1.15570 \\
\hline$\Gamma_{16}$ & 6.06961 & 120.360 \\
\hline
\end{tabular}

\section{Estimates of gluon concentrations}

To obtain necessary estimates we shall use an analogy with classical electrodynamics where is well known (see e. g. [10]) that the notion of classical electromagnetic field (a photon condensate) generated by a charged particle is applicable only at distances $>>$ the Compton wavelength $\lambda_{c}=1 / m$ for the given particle. If denoting $\lambda_{B}$ the de Broglie wavelength of the particle then $\lambda_{B}=1 / p$ with the relativistic impulse $p=m v / \sqrt{1-v^{2}}$ while $v$ is the velocity of the particle (as a result, $\lambda_{c}=\lambda_{B}$ at $v=1 / \sqrt{2}$ ) so one can rewrite $\lambda_{B}=\lambda_{c} \sqrt{1-v^{2}} / v$ and it is clear that $\lambda_{B} \rightarrow 0$ when $v \rightarrow 1$ (ultrarelativistic case), i. e., the particle becomes more and more point-like one. Accordingly, one can conclude that in the latter case the notion of classical electromagnetic field generated by a charged ultrarelativistic particle is applicable at distances $>>\lambda_{B}$. Under the circumstances, if the ultrarelativistic charged particle accomplishes its motion within the region with characteristic size of order $r_{0}$ then in the given region the electromagnetic field generated by the particle may be considered as classical one at $r_{0}>>\lambda_{B}$. For example, in the case of positronium we have $r_{0} \sim 2 a_{0}$, where $a_{0} \approx 5.29 \cdot 10^{-11} \mathrm{~m}$ is the Bohr radius, so $r_{0}>>\lambda_{e} \approx 3.86 \cdot 10^{-13} \mathrm{~m}$, the electron Compton wavelength, i. e., the electric Coulomb interaction between electron and positron in positronium can be considered as classical electromagnetic field.

Passing on to QCD, gluons and quarkonia, it should be noted that quarks in quarkonia accomplish a finite motion within a region of order $1 \mathrm{fm}=10^{-15} \mathrm{~m}$. Then, as is seen from the radial parts of the wave functions (13)-(14), the quantity $1 / \beta_{j}$ permits to be considered as a characteristic size of the $j$-th colour component of the given quarkonium state and, consequently, we can take the magnitude

$$
r_{0}=\frac{1}{3} \sum_{j=1}^{3} \frac{1}{\beta_{j}}
$$

for a characteristic size of the whole quarkonium state and, in line with the above, we should consider the confining SU(3)-gluonic Yang-Mills field of (3) or (6) to be classical one when $r_{0}>>\lambda_{B}$, the de Broglie wavelength of the corresponding quarks forming quarkonium.

On the other hand, a classical electromagnetic field (photon condensate) con- 
forms to the large photon concentrations for every frequency presented in the field [8]. Then in QCD we should require the large gluon concentrations in the given classical gluonic field (gluon condensate). To estimate the given concentrations we can employ $T_{00}$-component of the energy-momentum tensor of (5) and, taking the quantity $\omega=\Gamma$, the whole decay width of the quarkonium state, for the characteristic frequency we obtain the sought characteristic concentration $n$ in the form

$$
n=\frac{T_{00}}{\Gamma}
$$

It is not complicated to obtain the curvature matrix (field strentgh) corresponding to the solution (3) or (6)

$$
F=F_{\mu \nu}^{a} \lambda_{a} d x^{\mu} \wedge d x^{\nu}=-\partial_{r}\left(A_{t}^{a} \lambda_{a}\right) d t \wedge d r+\partial_{r}\left(A_{\varphi}^{a} \lambda_{a}\right) d r \wedge d \varphi
$$

which entails the only nonzero components

$$
F_{t r}^{3}=-\frac{2 a_{1}+a_{2}}{2 r^{2}}, F_{t r}^{8}=\frac{a_{2} \sqrt{3}}{2 r^{2}}, F_{r \varphi}^{3}=b_{1}+\frac{b_{2}}{2}, F_{r \varphi}^{8}=-\frac{b_{2} \sqrt{3}}{2}
$$

and, in its turn,

$$
\begin{aligned}
T_{00} \equiv T_{t t}=\frac{1}{4 \pi} & \left\{\frac{3}{4}\left[\left(F_{t r}^{3}\right)^{2}+\left(F_{t r}^{8}\right)^{2}\right]+\frac{1}{4 r^{2} \sin ^{2} \vartheta}\left[\left(F_{r \varphi}^{3}\right)^{2}+\left(F_{r \varphi}^{8}\right)^{2}\right]\right\}= \\
& \frac{3}{16 \pi}\left(\frac{a_{1}^{2}+a_{1} a_{2}+a_{2}^{2}}{r^{4}}+\frac{b_{1}^{2}+b_{1} b_{2}+b_{2}^{2}}{3 r^{2} \sin ^{2} \vartheta}\right),
\end{aligned}
$$

so, further putting $\sin ^{2} \vartheta=1 / 3$ for simplicity, we can rewrite (30) in the form

$$
T_{00}=T_{00}^{\text {coul }}+T_{00}^{\text {lin }}
$$

conforming to the contributions from the Coulomb and linear parts of the solution (3) or (6). The latter gives the corresponding split of $n$ from (27)

$$
n=n_{\text {coul }}+n_{\text {lin }}
$$

For comparison we shall also estimate the photon concentration in the ground state of the positronium. As is known historically [11], the analogy between the latter system and quarkonia played the important part when building the quarkonia models. For positronium we have the electromagnetic Coulomb interaction $A=A_{t} d t=(e / r) d t$ which entails $F=F_{t r} d t \wedge d r=\left(e / r^{2}\right) d t \wedge d r$ and

$$
T_{00} \equiv T_{t t}=\frac{1}{4 \pi}\left(F_{t r}\right)^{2}=\frac{\alpha_{e m}}{4 \pi r^{4}}
$$

with $\alpha_{e m}=e^{2}=1 / 137.0359895$. 


\section{Numerical results}

When computing for the first three states of charmonium we used their presentday whole decay widths $\Gamma=17.3 \mathrm{MeV}, 91.0 \mathrm{keV}, 10.2 \mathrm{MeV}$ respectively [5], while the calculation $r_{0}$ of (26) gives $r_{0}=r_{1}, r_{2}, r_{3}$ (see Table 4).

In the positronium case we employed the widths $\Gamma_{0}=1 / \tau_{0}$ (parapositronium) and $\Gamma_{1}=1 / \tau_{1}$ (orthopositronium), respectively, with the life times $\tau_{0}=1.252$. $10^{-10} \mathrm{~s}, \tau_{1}=1.377 \cdot 10^{-7} \mathrm{~s}[11]$ while $r_{0}=2 a_{0}$ with the Bohr radius $a_{0}=$ $0.529177249 \cdot 10^{5} \mathrm{fm}[5]$.

Tables 4, 5 contain the numerical results for both the cases, where we used $1 \AA=10^{-10} \mathrm{~m}=10^{5} \mathrm{fm}$. Finally, when calculating we applied the relation $1 \mathrm{GeV}^{-1} \approx 0.21030893 \mathrm{fm}$.

\section{Concluding remarks}

It is clear that for charmonium the natural unit of the gluon concentration is $\mathrm{fm}^{-3}$ while for positronium the photon concentration should be measured in $\AA^{-3}$. Then, as is seen from Tables 4, 5, qualitative behaviour of both the concentrations is similar. At the characteristic scales of each system the concentrations are large and the corresponding fields (electric and magnetic colour ones or electric Coulomb one) can be considered as classical ones. For charmonium the part $n_{\text {coul }}$ of gluon concentration $n$ connected with the Coulomb colour electric field is decreasing faster than $n_{\text {lin }}$, the part of $n$ related to the linear colour magnetic field, and at large distances $n_{\text {lin }}$ becomes dominant. Under the circumstances, as has been said in Section 4, we can estimate the quark velocities in the charmonium states under discussion from the condition

$$
v=\frac{1}{\sqrt{1+\left(\frac{\lambda_{B}}{\lambda_{q}}\right)^{2}}}
$$

with the $c$-quark Compton wavelength $\lambda_{q}=1 /\left(2 \mu_{0}\right) \approx 0.168247 \mathrm{fm}$ so, taking the de Broglie wavelength $\lambda_{B}=0.1 r_{k}(k=1,2,3)$ with $r_{k}$ from Table 4, we obtain $v_{1} \approx 0.999718, v_{2} \approx 0.999721, v_{3} \approx 0.999715$ i. e., the quarks in charmonium should be considered as the ultrarelativistic point-like particles. This additionally confirms the conclusion of Refs. $[2,3]$ that the relativistic effects are extremely important for the confinement mechanism. As a result, the confinement scenario described early in Section 1 may really occur.

The results of the given paper can be extended to both other states of charmonium and the bottomonium states. We hope to return to the problem else- 
Table 4

Gluon concentrations in the first three states of charmonium.

\begin{tabular}{cccc}
$\eta_{c}(1 S):$ & $r_{1}=0.0399766 \mathrm{fm}$ & & \\
\hline$r$ & $n_{\text {coul }}$ & $n_{\text {lin }}$ & $n$ \\
$\mathrm{fm}$ & $\left(\mathrm{fm}^{-3}\right)$ & $\left(\mathrm{fm}^{-3}\right)$ & $\left(\mathrm{fm}^{-3}\right)$ \\
\hline
\end{tabular}

\begin{tabular}{cccc}
$0.1 r_{1}$ & $0.550649 \cdot 10^{13}$ & $0.727630 \cdot 10^{10}$ & $0.551377 \cdot 10^{13}$ \\
$r_{1}$ & $0.550649 \cdot 10^{9}$ & $0.727630 \cdot 10^{8}$ & $0.623412 \cdot 10^{9}$ \\
$10 r_{1}$ & $0.550649 \cdot 10^{5}$ & $0.727630 \cdot 10^{6}$ & $0.782695 \cdot 10^{6}$ \\
1.0 & $0.140637 \cdot 10^{4}$ & $0.116285 \cdot 10^{6}$ & $0.117691 \cdot 10^{6}$ \\
$a_{0}$ & $0.179347 \cdot 10^{-15}$ & $0.415260 \cdot 10^{-4}$ & $0.415260 \cdot 10^{-4}$ \\
\hline
\end{tabular}

$J / \psi(1 S): \quad r_{2}=0.0397797 \mathrm{fm}$

\begin{tabular}{cccc}
$r$ & $n_{\text {coul }}$ & $n_{\text {lin }}$ & $n$ \\
$\mathrm{fm}$ & $\left(\mathrm{fm}^{-3}\right)$ & $\left(\mathrm{fm}^{-3}\right)$ & $\left(\mathrm{fm}^{-3}\right)$ \\
\hline
\end{tabular}

\begin{tabular}{cccc}
$0.1 r_{2}$ & $0.106772 \cdot 10^{16}$ & $0.139702 \cdot 10^{13}$ & $0.106912 \cdot 10^{16}$ \\
$r_{2}$ & $0.106772 \cdot 10^{12}$ & $0.139702 \cdot 10^{11}$ & $0.120742 \cdot 10^{12}$ \\
$10 r_{2}$ & $0.106772 \cdot 10^{8}$ & $0.139702 \cdot 10^{9}$ & $0.150380 \cdot 10^{9}$ \\
1.0 & $0.267364 \cdot 10^{6}$ & $0.221069 \cdot 10^{8}$ & $0.223742 \cdot 10^{8}$ \\
$a_{0}$ & $0.340956 \cdot 10^{-13}$ & $0.789450 \cdot 10^{-2}$ & $0.789450 \cdot 10^{-2}$ \\
\hline
\end{tabular}

$\chi_{c 0}(1 P): \quad r_{3}=0.0401851 \mathrm{fm}$

\begin{tabular}{cccc}
$r$ & $n_{\text {coul }}$ & $n_{\text {lin }}$ \\
$\mathrm{fm}$ & $\left(\mathrm{fm}^{-3}\right)$ & $\left(\mathrm{fm}^{-3}\right)$ & $\begin{array}{c}n \\
\left(\mathrm{fm}^{-3}\right)\end{array}$ \\
\hline & & & \\
$0.1 r_{3}$ & $0.914712 \cdot 10^{13}$ & $0.122134 \cdot 10^{11}$ & $0.915933 \cdot 10^{13}$ \\
$r_{3}$ & $0.914712 \cdot 10^{9}$ & $0.122134 \cdot 10^{9}$ & $0.103685 \cdot 10^{10}$ \\
$10 r_{3}$ & $0.914712 \cdot 10^{5}$ & $0.122134 \cdot 10^{7}$ & $0.131282 \cdot 10^{7}$ \\
1.0 & $0.238531 \cdot 10^{4}$ & $0.197228 \cdot 10^{6}$ & $0.199613 \cdot 10^{6}$ \\
$a_{0}$ & $0.304186 \cdot 10^{-15}$ & $13.704313 \cdot 10^{-4}$ & $0.704313 \cdot 10^{-4}$ \\
\hline
\end{tabular}


Table 5

Photon concentrations in the ground state of positronium.

$$
r_{0}=2 a_{0}=2 \cdot 0.529177249 \cdot 10^{5} \mathrm{fm}
$$

\begin{tabular}{ccc} 
& Parapositronium & Orthopositronium \\
$r$ & $n_{\text {para }}$ & $n_{\text {ortho }}$ \\
$\mathrm{fm}$ & $\left(\mathrm{fm}^{-3}\right.$ or $\left.\AA^{-3}\right)$ & $\left(\mathrm{fm}^{-3}\right.$ or $\left.\AA^{-3}\right)$ \\
\hline & & \\
$0.01 r_{0}$ & $0.888025 \cdot 10\left(0.888025 \cdot 10^{16}\right)$ & $0.976685 \cdot 10^{4}\left(0.976685 \cdot 10^{19}\right)$ \\
$0.1 r_{0}$ & $0.888025 \cdot 10^{-3}\left(0.888025 \cdot 10^{12}\right)$ & $0.976685\left(0.976685 \cdot 10^{15}\right)$ \\
$r_{0}$ & $0.888025 \cdot 10^{-7}\left(0.888025 \cdot 10^{8}\right)$ & $0.976685 \cdot 10^{-4}\left(0.976685 \cdot 10^{11}\right)$ \\
$2 r_{0}$ & $0.555015 \cdot 10^{-8}\left(0.555015 \cdot 10^{7}\right)$ & $0.610428 \cdot 10^{-5}\left(0.610428 \cdot 10^{10}\right)$ \\
\hline
\end{tabular}

where.

\section{References}

[1] Yu. P. Goncharov, Mod. Phys. Lett. A 16 (2001) 557.

[2] Yu. P. Goncharov and E. A. Choban, Mod. Phys. Lett. A 18 (2003) 1661.

[3] Yu. P. Goncharov, Europhys. Lett. 62 (2003) 684.

[4] Yu. P. Goncharov, Pis'ma v ZhETF 69 (1999) 619;

Phys. Lett. B 458 (1999) 29.

[5] S. Eidelman et al. (Particle Data Group), Phys. Lett. B 592 (2004) 1.

[6] J. H. Kühn and M. Steinhauser, Nucl. Phys. B 619 (2001) 588; Erratum-ibid. B 640 (2002) 415;

V. G. Krivokhijine and A. V. Kotikov, Acta Phys. Slov. 52 (2002) 227; Acta Phys. Polon. B 33 (2002) 2947; Nucl. Instrum. Meth. A 502 (2003) 624;

ALEPH Collaboration, Eur. Phys. J. C 27 (2003) 1.

[7] R. Bonnaz, B. Silvestre-Brac and C. Gignoux, Eur. Phys. J. A 13 (2002) 363.

[8] V. B. Berestezkiy, E. M. Lifshits and L. P. Pitaevskiy, Relativistic Quantum Theory (Nauka, Moscow, 1968). 
[9] A. P. Prudnikov, Yu. A. Brychkov and O. I. Marichev, Integrals and Series. Elementary Functions (Nauka, Moscow, 1981).

[10] L. D. Landau and E. M. Lifshits, Field Theory (Nauka, Moscow, 1967).

[11] D. H. Perkins, Introduction to High Energy Physics (Addison-Wesley Publishing Company, Inc., London, 1987) 